\title{
Interactions between spherical colloids mediated by a liquid crystal: A molecular simulation and mesoscale study
}

\author{
Evelina B. Kim, Orlando Guzmán, Sylvain Grollau, Nicholas L. Abbott, \\ and Juan J. de Pablo \\ Department of Chemical and Biological Engineering, University of Wisconsin, Madison, Wisconsin 53706
}

(Received 18 March 2004; accepted 21 April 2004)

\begin{abstract}
Monte Carlo simulations and dynamic field theory (DyFT) are used to study the interactions between dilute spherical particles, dispersed in nematic and isotropic phases of a liquid crystal. A recently developed simulation method (expanded ensemble density of states) was used to determine the potential of mean force (PMF) between the two spheres as a function of their separation and size. The PMF was also calculated by a dynamic field theory that describes the evolution of the local tensor order parameter. Both methods reveal an overall attraction between the colloids in the nematic phase; in the isotropic phase, the overall attraction between the colloids is much weaker, whereas the repulsion at short range is stronger. In addition, both methods predict a new topology of the disclination lines, which arises when the particles approach each other. The theory is found to describe the results of simulations remarkably well, down to length scales comparable to the size of the molecules. At separations corresponding to the width of individual molecular layers on the particles' surface, the two methods yield different defect structures. We attribute this difference to the neglect of density inhomogeneities in the DyFT. We also investigate the effects of the size of spherical colloids on their interactions. (C) 2004 American Institute of Physics.
\end{abstract}

[DOI: 10.1063/1.1761054]

\section{INTRODUCTION}

Besides their traditional use in displays and thermography, liquid crystals (LCs) are also finding applications in biomolecular sensors. ${ }^{1,2}$ This type of biosensor is essentially a thin cell containing a nematic fluid. The cell's walls have receptors for specific biomolecules and, in the absence of binding, the wall chemistry and topography impose a uniform orientation of the LC throughout the cell. The sensor is able to detect the binding of biomolecules at the walls because they induce topological defects that destroy the uniform orientation texture. A nematic exhibits characteristic defects known as disclination lines. They can be identified as extended discontinuities in the director field, that is, lines where the average molecular orientation changes abruptly (over a few molecular lengths). ${ }^{3}$ Since nematics possess long-range orientational order, the defects associated to binding events at the nanoscopic level are "amplified" to the millimeter length scale: a pair of crossed polarizers and a microscope are sufficient to pick up the optical signature of the disclination lines.

A fundamental and detailed understanding of the operation of the sensor is important for the design of new, optimized versions. However, the processes at work involve multiple length scales and this represents a challenge if one expects to obtain an accurate representation of the system directly from the smallest scales. As an example of the different processes that need to be modeled, we can mention the anchoring of the LC to the sensor walls (operating at nanoscopic scale), the propagation of the uniform orientation induced by a given anchoring condition into the bulk of the cell (mesoscopic), and the relaxation of the defect structures over relatively long time scales.

As our long-term goal is to model accurately the biosensor system, we have adopted a multiscale approach to this problem. We use molecular simulations (MSs) to explore the system at the shorter scale $(\mathrm{nm})$, while a dynamic field theory (DyFT) is employed to study the system at longer scales $(\mu \mathrm{m})$. In a model of a real sensor, the problem will require that many nanoscopic colloids be distributed over macroscopic distances; molecular simulations would not be effective and we will have to resort to a coarse-grained model (that is, DyFT). We have found it advantageous to analyze a sequence of systems of increasing complexity: starting from systems with a few colloidal particles and then moving to systems with many colloids.

In this paper we analyze a two-colloid system embedded in a LC-host using both molecular simulations and dynamic field theory. Our purpose is twofold: the first is to test correspondence between MS and DyFT, finding length and energy scale mappings between them, and establishing the range of validity of the theory. The second is to investigate the twoparticle system's effective interactions, its defect structures and density and orientational profiles. In this regard, we also explore the effects of varying the colloids size, and of replacing the nematic host with an isotropic liquid.

We note that here the particles are suspended in the interior of a LC cell, whereas in a sensor they are adsorbed on a substrate. Our choice is driven by simplicity (purely twobody colloidal interactions, where the walls provide a specified bulk director field) and relevance to the controlled ex- 
periments with colloidal particles suspended in LC discussed below. The investigation of this system is also relevant to design of sensors where the particles to be detected would be adsorbed from the bulk LC. Studies of a biosensor system employing DyFT with particles adsorbed on the walls are currently in progress and will be published elsewhere.

Systems of LCs and one or two colloids have been studied by experimental, ${ }^{4-8}$ simulation, ${ }^{9-14}$ and theoretical methods ${ }^{15-24}$ in connection with their associated defect structures and effective interactions. The simplest case corresponds to a single spherical colloid embedded in a nematic fluid, with strong homeotropic (perpendicular) anchoring at the colloid surface. For this system, the defect structures that have been identified by the above methods are the hyperbolic point defect (also known as hedgehog), the equatorial Saturn ring line defect, and intermediate off-equator rings. For large (micron-sized) particles in bulk nematics, only the hedgehog defect is stable. Conversely, for small, nanoscopic particles the situation is reversed and the Saturn ring becomes stable. The Saturn ring configuration can also be stabilized for micron-sized particles by confinement. ${ }^{4,23}$ In a previous paper, ${ }^{13}$ we have presented our results from simulation and field theory for a one-colloid system confined between parallel walls, including density and orientational profiles, structure of the Saturn ring defect, and the potential of mean force (PMF) between the colloid and the cell's walls.

Systems of two colloids immersed in solutions of rods have been studied experimentally by Verma et $a l^{7}$ and Lin et $a l^{8}{ }^{8}$ They measured the potential of mean force between colloids in solutions of DNA or fd-virus, using optical tweezers methods. The anisotropic particles used by them have an aspect ratio (that is, the ratio of a rod's length to its diameter) on the order of one hundred; in order to operate the tweezers, the concentration of the solutions must be in the isotropic regime. An alternative approach to the optical tweezers method was used by Poulin et al. for less elongated molecules in order to measure the effective forces between colloids in the anisotropic environment of the nematic phase. ${ }^{5}$ They prepared droplets of ferromagnetic fluid inside a nematic sample, used a small magnetic field to drive the droplets apart, and then track their position as a function of time: from this information the effective force acting on the droplets was inferred.

From the theoretical standpoint, Galatola and co-workers ${ }^{21,22,24}$ have performed analytical calculations within the framework of dynamic field theory of the tensor order parameter ${ }^{25} \mathbf{Q}$. Compared to the continuum theory for the director, ${ }^{3}$ the DyFT for $\mathbf{Q}$ is free of divergences and the equations of motion for the tensor components are simpler. For a two-colloid system in the nematic phase, the DyFT calculations must be performed numerically. Stark and coworkers have used this approach to study pairs of droplets immersed in a spherically confined nematic. ${ }^{17}$ Using finite element methods, they computed the potential of mean force and director profiles associated with different sphere configurations with cylindrical symmetry. More recently, Tasinkevych et al. have used this same theory in the case of bidimensional systems with two infinitely long cylinders dispersed in a nematic, performing both an approximate analytical treatment of the problem and finite-element calculations. ${ }^{20}$ They concluded that at small separations the defects associated with each cylinder may change their positions, leading to an unexpected reorganization of the interaction energies. In this paper we present evidence that, in three dimensions, the disclination lines that arise around spherical colloids do not only change their positions, but also are able to reorganize their topology when the particles are very close to each other. An abbreviated version of our findings has been presented in the literature. ${ }^{14}$ In this work we provide a more extensive and detailed discussion of such systems.

The paper is organized as follows. In Sec. II we describe the models and procedures used to simulate the system under study. In particular, we describe briefly the expanded ensemble density of states (ExEDOS) Monte Carlo method used in this and previous work. ${ }^{12,13}$ The ExEDOS method enables one to simulate the system efficiently. To the best of our knowledge, no results for this type of systems have been reported by any other molecular simulation method. Next, a description of the dynamic field theory approach is provided in Sec. III. Section IV is devoted to the results (of MS and DyFT) and its discussion. It is divided in three parts: Sec. IV A, presenting the PMF for the colloids in the nematic phase, as well as an analysis of the defect structures associated with the spheres; Sec. IV B, comparing the PMF obtained in the nematic to those obtained in the isotropic phase; and Sec. IV C, discussing the effects of the colloids' size on their effective interaction. We finish with concluding remarks in Sec. V.

\section{MONTE CARLO SIMULATIONS}

\section{A. Expanded ensemble density of states method}

We utilize a recently proposed ExEDOS simulation method $^{12,26}$ to simulate the system's behavior as a function of the colloid's separation. In general terms, the method combines the expanded ensemble technique with the powerful density of states formalism. The latter is advantageous for updating on the fly the set of weights required by the former, instead of having to compute them iteratively at the end of many regular expanded ensemble runs. This set of weights is directly related to the PMF of the system. In this sense, ExEDOS offers efficient sampling of the PMF as opposed to the alternative method of simulating a system at a series of fixed separations.

The PMF is defined (in general) as the free energy of the system as a function of one or several degrees of freedom, which are often referred to as "reaction coordinate" $\zeta$. In the present case of two spheres suspended at the middle of the cell, there is only one degree of freedom that corresponds to the separation between the spheres. When the spheres are embedded in a nematic phase, there is an additional degree of freedom corresponding to the angle between the line connecting spheres' centers of mass and the bulk director; in this paper this angle is set to $90^{\circ}$.

The PMF can be interpreted as the amount of work required to bring two spheres from infinity to a specified distance $d$ and is equal to the difference in the free energy of the 
a)

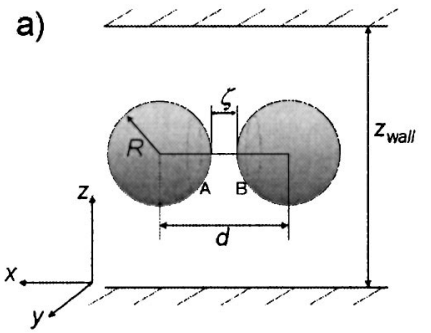

b)

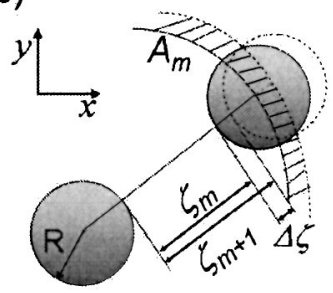

FIG. 1. (a) Schematic view of the system. (b) Definition of the expanded states.

system with the two spheres at distance $d$ and at infinite separation,

$$
w(d)=F(d)-F(\infty) .
$$

We have successfully applied the ExEDOS method in the calculations of the PMF between a single colloid and a planar wall. The ExEDOS algorithm remains unchanged and, therefore, we refer the reader to Ref. 12 for a detailed description of the method. Here, instead, we define relevant variables and the expanded states; we also highlight differences in the application of the method for the present application and the sphere/substrate case.

The system under consideration contains $N$ mesogens confined by two soft repulsive walls placed at $z=0$ and $z$ $=Z_{\text {wall }}$. Two soft repulsive spheres of radius $R$ are free to move with their centers of mass restricted to the plane $z$ $=Z_{\text {wall }} / 2$. The centers of mass of the spheres are separated by a distance $d$; the closest approach is $2 R$ and so we introduce as reaction coordinate

$$
\zeta=d-2 R,
$$

which is the separation between the spheres' surfaces [as indicated in Fig. 1(a)].

In accordance with the expanded ensemble technique, we discretize or expand in the reaction coordinate $\zeta$ by defining a set of $M$ intervals of length $\Delta \zeta$ :

$$
[(m-1) \Delta \zeta, m \Delta \zeta), m=1, M .
$$

The system is said to be in a state $m$ if $(m-1) \leqslant \zeta / \Delta \zeta<m$ [see Fig. 1(b)]. For sufficiently short intervals, we can associate with each state the midpoint $\zeta_{m}=(m-1 / 2) \Delta \zeta$ as the representative value of the state.

The partition function for the entire system of spheres and LC (in a volume $V$ at temperature $T$ ) is

$$
\Omega=\sum_{m=1}^{M} Q(N, V, T, m) g_{m}=\sum_{m=1}^{M} Q_{m} g_{m},
$$

where $Q_{m}$ and $g_{m}$ designate a canonical partition function and a weighting factor for a state $m$, respectively. The former is the quantity of interest, since we can obtain the PMF from it as

$$
\beta w_{m}(\xi)=-\ln Q_{m}+C,
$$

where $\beta=1 / k T$ and $C$ is a constant independent of $m$.

The core of the ExEDOS method is to update the estimate of the weighting factors $g_{m}$ by an amount $\ln f$ every time a state is visited ( $f$ is called the convergence factor),

while achieving a flat histogram of visits to each state; this procedure is iterated for decreasing values of $\ln f$. It is important to note that the histogram has to be normalized by the area $A_{m}=2 \pi\left(2 R+\zeta_{m}\right) \Delta \zeta$ associated with the state $m$ in the plane $z=Z_{\text {wall }} / 2$ as indicated in Fig. 1(b). This accounts for the fact that there are more conformations of two spheres at larger separations. In ordinary density of states simulations, where a random walk in energy is performed, the threshold convergence factor is set to $\ln f=10^{-7}$ or $\ln f=10^{-8}$ in order to achieve acceptable accuracy for the density of energy states; in this application much less stringent thresholds are sufficient for obtaining acceptable estimates of the weights for the separation states. In particular, in this work we use threshold values ranging between $\ln f=5 \times 10^{-5}$ and $\ln f=4$ $\times 10^{-4}$, which correspond to 9-12 successive updates of the convergence factor.

One of the benefits of using the ExEDOS method is that, in addition to the PMF estimated from the weights, another estimate can be obtained by measuring and subsequently integrating the mean force on each sphere at each state, $F_{\text {sph }}\left(\zeta_{m}\right)$ :

$$
w\left(\zeta_{m}\right)-w\left(\zeta_{M}\right)=-\int_{\zeta_{m}}^{\zeta_{M}} F_{\mathrm{sph}}(\zeta) d \zeta .
$$

The force $F_{\text {sph }}\left(\zeta_{m}\right)$ is a simple canonical average of the total force on either sphere along the interparticle vector, due to its interactions with the LC molecules. The forces on two spheres must be, on average, of equal magnitude and opposite sign; we use the average of the magnitude for the two spheres.

The two independent estimates of the PMF, $w\left[g_{m}\right]$ [Eq. (5)] and $w\left[F_{\mathrm{sph}}\left(\zeta_{m}\right)\right]$ [Eq. (6)] must agree when the set of weights has converged; therefore, in addition to the condition $f \leqslant f_{\text {thr }}$, the agreement between these two estimates should be another requirement in the criterion for convergence of the simulation. After the agreement has been found, either estimate of the PMF can be used. In this work, we report the PMF obtained by integration of the mean force as these curves have a smoother appearance than those obtained from the weights.

\section{B. Simulation details}

The system under study comprises 11460 (12718 for the larger sphere) liquid crystal particles confined between two soft repulsive walls at $z=0$ and $z=Z_{\text {wall }}$. The LC molecules are represented by soft repulsive ellipsoids of revolution having a length-to-width $\left(\sigma_{0}\right)$ ratio $\kappa$ of 3. Each molecule $\alpha$ is characterized by a center of mass position vector $\mathbf{r}_{\alpha}$ and an orientation unit vector $\hat{\mathbf{u}}_{\alpha}$. Mesogens interact with themselves, the walls, and the spheres via a shifted and truncated Gay-Berne potential, ${ }^{12}$ while spheres interact with each other as hard bodies. In this work, the separation between the walls was set at $Z_{\text {wall }}=34 \sigma_{0}$ for spheres of radius of $3 \sigma_{0}$ and $Z_{\text {wall }}=39 \sigma_{0}$ for spheres of radius of $5 \sigma_{0}$. The choice of $Z_{\text {wall }}$ was dictated by the width of the bulk region of the film, i.e., the region where the layered structure of LC induced by the walls was absent. 
The interaction potentials described above result in homeotropic (perpendicular) anchoring of LC molecules at all interfaces. Homeotropic and planar anchoring of varying strength $W$ could be introduced into the simulations by incorporating interactions that depend on the orientation of the mesogens relative to the confining surfaces. ${ }^{9}$ For a typical value $K$ of the elastic constants, one expects weak anchoring behavior $^{16}$ if $W / K R \ll 1$. A description of possible defect structures for arbitrary anchoring conditions is beyond the scope of this paper, and we limit our attention to the strong anchoring case. We note that the anchoring of a typical LC [such as pentylcyanobiphenyl $(5 \mathrm{CB})]$ on viruses with lipid envelopes is generally strong and homeotropic. ${ }^{27}$

For the estimation of the mean force acting on colloids, the total force on a sphere is calculated as the sum of the forces between the mesogens and the sphere; $F_{\mathrm{sph}}$ is defined as the projection of the total force on the line connecting the CMs of the two spheres.

Simulations were conducted at a constant reduced temperature $T^{*}=k T / \epsilon_{0}=1.0$ and an average reduced number density of $\rho^{*}=N \sigma_{0}^{3} / V=0.335$ in the nematic phase and $\rho^{*}=0.300$ in the isotropic phase; $\sigma_{0}$ and $\epsilon_{0}$ are length and energy parameters. The simulation box is rectangular with the side lengths equal in $x$ and $y$ directions. Periodic boundary conditions apply in the $x$ and $y$ directions.

The ExEDOS simulations were conducted for spheres of $R=3 \sigma_{0}$ in the isotropic and nematic phases. The range of the reaction coordinate $d$ varied from the lower boundary of $2 R$ (spheres at contact) to $d=15 \sigma_{0}$ (no interaction). This range is rather large if the separation between the states, $\Delta \zeta$, is to be small for accurate integration of the mean force. For our choice of $\Delta \zeta$ of $0.01 \sigma_{0}$ we divided the entire range of the reaction coordinate into fully overlapping windows of width $2 \sigma_{0}$ i.e., each window hosts $M=200$ states. A move to a new state, i.e., displacement of a sphere within the $x y$ plane is attempted once per sphere every Monte Carlo cycle; a cycle consists of all liquid crystal particles having been attempted to either be displaced or rotated once.

In order to extract static properties of the system, such as number density and second-rank order parameter $P_{2}$ (also denoted by $S$ ), we performed a series of separate $N V T$ ensemble simulations with spheres fixed at selected values of $\zeta$. These simulations were run for $1 \times 10^{6} \mathrm{MC}$ cycles and trajectories were recorded every 2000 cycles. In the analysis of thus collected trajectories, LC molecules were binned according to a three-dimensional rectangular grid and properties of interest were calculated for each bin. $P_{2}$ was taken as $3 / 2$ of largest eigenvalue of the order parameter tensor $\mathbf{Q}$ calculated from the orientations $\hat{\mathbf{u}}_{\alpha}$ of $N_{V}$ particles in a bin volume $d \mathbf{r}$ as

$$
\mathbf{Q}=\frac{1}{N_{V}} \sum_{\alpha=1}^{N_{V}}\left(\hat{\mathbf{u}}^{\alpha} \hat{\mathbf{u}}^{\alpha}-\frac{1}{3} \mathbf{I}\right),
$$

where I denotes an identity tensor. The eigenvector $\mathbf{n}$ associated with $P_{2}$ is the director or the average orientation of $N_{V}$ particles.

\section{DYNAMIC FIELD THEORY FOR THE TENSOR ORDER PARAMETER}

This approach is based on coarse graining the distribution of molecular orientations into a second-rank tensor order parameter $\mathbf{Q}(\mathbf{r})$ and applying the bracket formalism developed by Beris and Edwards ${ }^{25}$ to the field $\mathbf{Q}(\mathbf{r})$.

Within the assumption of a spatially uniform density, an anisotropic fluid can be described by the orientational probability distribution function, $f(\mathbf{r}, \hat{\mathbf{u}})$, where $\hat{\mathbf{u}}$ is an orientation of the molecular axis. The detailed information contained in $f(\mathbf{r}, \hat{\mathbf{u}})$ can be coarse grained to a mesoscopic field, represented by the tensor of second moments of $f$ :

$$
\mathbf{Q}_{i j}=\int\left(u_{i} u_{j}-\frac{1}{3} \delta_{i j}\right) f(\mathbf{r}, \hat{\mathbf{u}}) d \hat{\mathbf{u}} .
$$

Here $i$ and $j$ refer to the components of an orientation unit vector $\hat{\mathbf{u}}$; the customary summation over repeated indices is assumed. This definition is equivalent to Eq. (7) where the order parameter tensor is written in terms of molecular orientations $\hat{\mathbf{u}}^{\alpha}$ contained in a sample volume. Either definition renders $\mathbf{Q}(\mathbf{r})$ a symmetric traceless second-rank tensor. In general, it can be diagonalized and written in the form

$$
\mathbf{Q}=\left(\begin{array}{ccc}
\frac{2 P_{2}}{3} & 0 & 0 \\
0 & \frac{\eta-P_{2}}{3} & 0 \\
0 & 0 & -\frac{\eta+P_{2}}{3}
\end{array}\right),
$$

where $P_{2}$ denotes the nematic scalar order parameter and $\eta$ denotes the biaxiality. The eigenvector associated with the highest eigenvalue $2 \mathrm{P}_{2} / 3$ corresponds to the director $\mathbf{n}$.

The free energy density $\mathcal{F}[\mathbf{Q}(\mathbf{r})]$ can now be expressed in terms of $\mathbf{Q}$ and its derivatives or, in other words, the short-range, $\mathcal{F}_{s}$, and long-range, $\mathcal{F}_{e}$, contributions, respectively. The short-range part is represented by a Landau-de Gennes power series expansion, ${ }^{3}$

$$
\begin{aligned}
\mathcal{F}_{s}= & \int\left\{\frac{A}{2}\left(1-\frac{U}{3}\right) Q_{i j} Q_{i j}-\frac{A U}{3} Q_{i j} Q_{i k} Q_{k j}\right. \\
& \left.+\frac{A U}{4}\left(Q_{i j} Q_{i j}\right)^{2}\right\} d \mathbf{r},
\end{aligned}
$$

and reflects the excluded volume effects that are responsible for the first-order isotropic-nematic transition. $A$ and $U$ are phenomenological coefficients that nevertheless can be given a microscopic interpretation. ${ }^{25}$ In general, the nematic potential $U$ can be assigned dependence on either concentration or temperature depending on the lyotropic or thermotropic nature of a liquid crystal. In the model used here, the fluid is isotropic for $U<2.7$ and it is nematic otherwise.

For an adequate free energy description of inhomogeneities in the $\mathbf{Q}$ field, one must account for the gradients of $\mathbf{Q}$. That is done in the long range elastic contribution to $\mathcal{F}$ :

$$
\mathcal{F}_{e}=\int d \mathbf{r} \frac{L_{1}}{2}\left(\partial_{i} Q_{j k}\right)\left(\partial_{i} Q_{j k}\right),
$$


where $L_{1}$ is a material specific elastic coefficient. In one constant approximation $L_{1}$ is related to the splay $K_{11}$, bend $K_{22}$, and twist $K_{33}$ constants by $2 L_{1}=K_{11} / S^{2}=K_{22} / S^{2}$ $=K_{33} / S^{2}$. In the above equations, $A, U$, and $L_{1}$ constitute a set of phenomenological parameters that naturally arise in the theory. From the dimensional analysis one can deduce a characteristic length scale of the model, namely, a coherence length $^{28} \xi=\sqrt{18 L_{1} / A U}$, defined as a distance over which the order parameter changes or a thickness of a domain wall. Both, $\xi$ and $L_{1}$ can be obtained experimentally-in this work we chose these parameters to correspond to the physical properties of $5 \mathrm{CB} .{ }^{29}$ The choice of the nematic potential will dictate the bulk value of the scalar order parameter. Parameter $A$, which is a common factor in all the terms comprising the short range free energy $\mathcal{F}_{s}$ and whose units are those of the free energy density, serves as the parameter controlling the relative contributions of $\mathcal{F}_{s}$ and $\mathcal{F}_{e}$ to the total free energy, $\mathcal{F}=\mathcal{F}_{s}+\mathcal{F}_{e}$, i.e., $\mathcal{F}$ can be measured in units of $A$.

The time evolution of the field $\mathbf{Q}$ is governed by the functional derivative of the free energy with respect to $\mathbf{Q}$ :

$$
\frac{\partial \mathbf{Q}}{\partial t}=\Gamma\left(-\frac{\delta \mathcal{F}}{\delta \mathbf{Q}}+\frac{\mathbf{I}}{3} \operatorname{Tr} \frac{\delta \mathcal{F}}{\delta \mathbf{Q}}\right),
$$

with I being the identity operator. The last term in Eq. (12) guarantees that the order parameter tensor $\mathbf{Q}$ is traceless at the minimum free energy. The proportionality coefficient $\Gamma$ is related to the rotational diffusivity $D^{*}$ through $\Gamma=6 D^{*} /(1$ $\left.-3 / 2 Q_{m n} Q_{m n}\right)^{2}$. Upon substitution of this expression for $\Gamma$ into Eq. (12), one obtains the equation of motion of the field $\mathbf{Q}$ that can be solved numerically using, for example, a finite difference method (as we do here). Boundary conditions are determined by the LC anchoring on all interfaces present in the system of interest. In this study, we restrict ourselves to the case of strong homeotropic anchoring exhibited by all bounding surfaces, i.e., walls and spheres. At the boundaries we may assume that $\mathbf{Q}$ is uniaxial and has the form of Eq. (9) with the biaxiality $\eta$ set to zero:

$$
\mathbf{Q}=P_{2}^{\mathrm{eq}}\left(\mathbf{n n}-\frac{1}{3} \mathbf{I}\right)
$$

where $\mathbf{n}$ is the director at the surface and $P_{2}^{\mathrm{eq}}$ is the equilibrium nematic order parameter given by ${ }^{30}$

$$
P_{2}^{\mathrm{eq}}=\frac{1}{4}+\frac{3}{4} \sqrt{1-\frac{8}{3 U}} .
$$

In order to make a direct comparison with the results of molecular simulations, we set $U=4.8(U=1.0)$, which corresponds to $P_{2}^{\mathrm{eq}}$ of $0.8(0.1)$ in the nematic (isotropic) bulk LC. ${ }^{31}$ The three-dimensional physical system is the same as in the molecular simulations (see Fig. 1) with periodic boundary conditions in the $x$ and $y$ directions. The spatial domain is discretized on a regular Cartesian grid which is defined everywhere except in the region inside the spheres. We verified the stability of the solution by employing various spatial mesh sizes and time steps; the present parameters are a $100 \times 100 \times 100$ mesh and $0.001 \leqslant \Delta t \leqslant 0.004$ time step range. The derivatives of the $\mathbf{Q}$ tensor are evaluated using the finite difference method while the integration of the con- stitutive equation is performed by applying an explicit Euler scheme. The initial configuration is that of a randomly oriented sample. We solve Eq. (12) for six components of the order parameter tensor, $Q_{x x}, Q_{y y}, Q_{z z}, Q_{x y}, Q_{x z}$, and $Q_{y z}$ over the entire spatial domain, ensuring that $\mathbf{Q}$ stays traceless.

\section{RESULTS AND DISCUSSION}

In the absence of microscopic experimental data, we have to rely on molecular simulations (MS) to obtain "exact" numerical results — exact for a given interaction potential with a provision of adequate phase space sampling. Therefore, it is our purpose to compare the MS results with those obtained from the field theory, highlighting those points where the two agree and those where the coarse graining fails. Such comparison will provide insight into how the theory could be improved and, at the same time, it will establish the range of validity for this particular field theory. In this view, for each feature of the system that we analyze, we provide first a description from a molecular point of view and then compare the situation with the theoretical picture.

In molecular simulations, a natural length scale is the molecular width $\sigma_{0}$ while in the theory, lengths may be measured in the units of the coherence length $\xi$. In order to obtain a mapping between these two scales, we need to identify some spatial feature of the system that is easy to compare in both the simulation and field-theoretical models. For this purpose we can use the radius $a$ of a Saturn ring accompanying a sphere of radius $R$ fixed at the midplane between the two walls. The idea is to match the curves of the ratio $a / R$ vs $R$ obtained by MS and DyFT, using a single proportionality constant $k^{L}=\xi / \sigma_{0}$. This same method was used in a previous publication, ${ }^{13}$ but in that instance using bidimensional DyFT data instead of three-dimensional (3D) calculations. In 3D, the defect forms closer to the sphere surface, thus increasing the proportionality constant between $\xi$ and $\sigma_{0}$. With the updated mapping, $\xi=7 \sigma_{0}$. In accordance with the new scaling, we performed 3D DyFT calculations and MS's for sphere sizes that correspond to $R$ of 3 and 5 units of $\sigma_{0}$. To investigate the effect of the size of colloids on their interactions, we computed additional PMFs by DyFT for colloid pairs of $R$ of up to $17.4 \sigma_{0}$.

\section{A. Colloidal interactions in the nematic phase}

We begin by discussing the effective interactions between the spheres, that is, their PMF. The MS results for the mean force and the PMF are summarized in Figs. 2 and 3, respectively, for spheres of radius $3 \sigma_{0}$ in the nematic phase; the corresponding results for the PMF calculated by the DyFT are shown in Fig. 4. Both forces and energies are plotted against the reaction coordinate $\zeta=d-2 R$.

First, we consider the MS results. As is apparent from the mean force and PMF plots, the overall interaction between two colloids is attractive. For very short distances, $\zeta$ $<1 \sigma_{0}$, spheres are strongly repeled, which is indicated by a positive mean force. Oscillations in the PMF as well as in the mean force in the attractive part of the potential can be attributed to the variation in density or, in other words, layering of liquid crystal molecules. In order to illustrate this and 


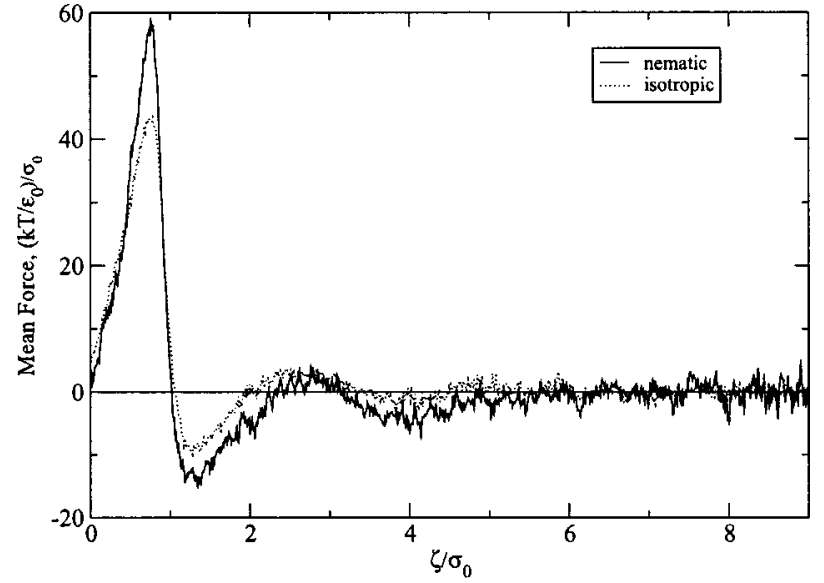

FIG. 2. Simulated mean force acting on the colloidal spheres of radius $R$ $=3 \sigma_{0}$ in the nematic and isotropic phases.

to understand the origin of the repulsive force, we examine the snapshots of the system at various separations shown in Fig. 5. At contact, the LC molecules found in the space between the spherical surfaces do not orient in the directions dictated by these surfaces due to lack of space. Instead, they arrange themselves in a flower-petals fashion orthogonally with respect to the vector d connecting the spheres' centers. This is illustrated in Fig. 5(a), where we show the director map superimposed on the density map.

As spheres move away from each other, more space is freed between them. The only way in which the LC molecules can satisfy approximately the anchoring conditions on both spherical surfaces is by being aligned with the vector $\mathbf{d}$. Hence, when the gap between the spheres is sufficiently large, the LC molecules indeed assume this preferred orientation and "push in" toward the line connecting the sphere centers. Thus a tube of horizontally aligned molecules is formed at $\zeta \approx 0.5 \sigma_{0}$ [see Fig. 5(b)]. As the molecules cannot penetrate all the way in the intersphere space, the tube is hollow; at $\zeta=1 \sigma_{0}$ a one-layer-long tube is completely filled

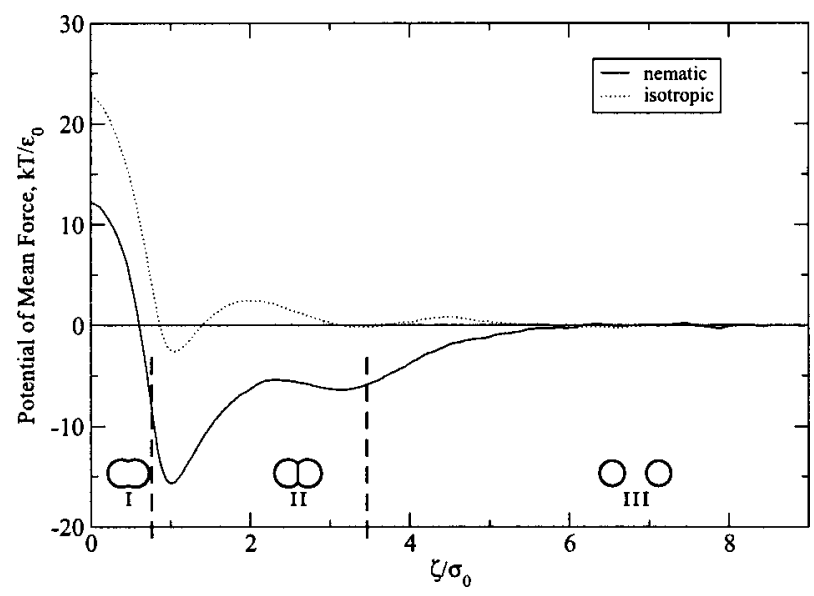

FIG. 3. Simulated potential of mean force between two spheres of radius $R=3 \sigma$ in the isotropic and nematic phases. Vertical dashed lines indicate the approximate location of the regions corresponding to a specified defect topology in the nematic phase.

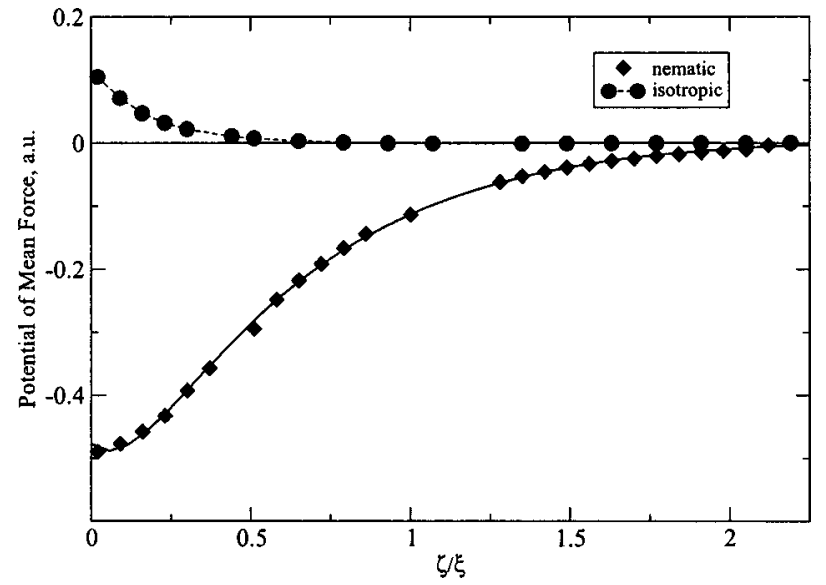

FIG. 4. Potential of mean force between two spheres in the isotropic and nematic phases for $R=0.62 \xi=3 \sigma_{0}$ by DyFT; the solid line corresponds to a polynomial fit of the PMF in the nematic phase, $a_{0}+a_{1} / d+a_{3} / d^{3}$ $+a_{5} / d^{5}$.

[see Fig. 5(c)]. At this point, the force ceases to be repulsive and the system reaches a global minimum in the free energy.

Further separation of the spheres causes diffusion of the molecular layer, thereby resulting in a (weak) attractive force. Consequently, the free energy increases, reaching a local maximum at $\zeta=2.2 \sigma_{0}$. The next energy minimum is reached when the space between the spheres accommodates exactly two layers of LCs-this takes place at $\zeta=3 \sigma_{0}$. The density contour maps for the separations corresponding to the three PMF extrema are shown in Fig. 6.

At this point it is instructive to make two remarks related to results that will be addressed later in this paper: first, note that, combined, two spheres of $R=3 \sigma_{0}$ can support a maximum of two layers with each layer corresponding to a local minimum in the PMF profile. Conceivably, the larger the spheres, the more layers they can support and, hence, the more minima will be apparent in the PMF. This is exactly the effect that we are able to observe when we increase the radius of the sphere to $5 \sigma_{0}$ in Sec. IV C. Second, the horizontal molecular orientation in the tube is in conflict with the vertical bulk orientation. This orientation discrepancy causes the formation of an additional disclination line not seen for a single sphere; we will analyze the defect structure later in this section.

It is also instructive to draw an analogy and to compare present results with those for a similar system explored in previous publications: ${ }^{13,32}$ there we studied the interactions mediated by LCs between a colloidal sphere and a wall. The similarity can be seen in that, in both cases, we have two homeotropically aligning interfaces and we seek to determine the interactions mediated by the LC molecules between these interfaces. The differences are that in the present situation, both interfaces are finite and spherical in shape, and, second, the bulk orientation of the LC is perpendicular to the line of interaction between the spheres. Despite the differences, many insights of the previous studies can be applied to the two-sphere system.

As in the sphere/substrate system, the force and the PMF curves shown in Fig. 2 (and Fig. 3) display an oscillatory 

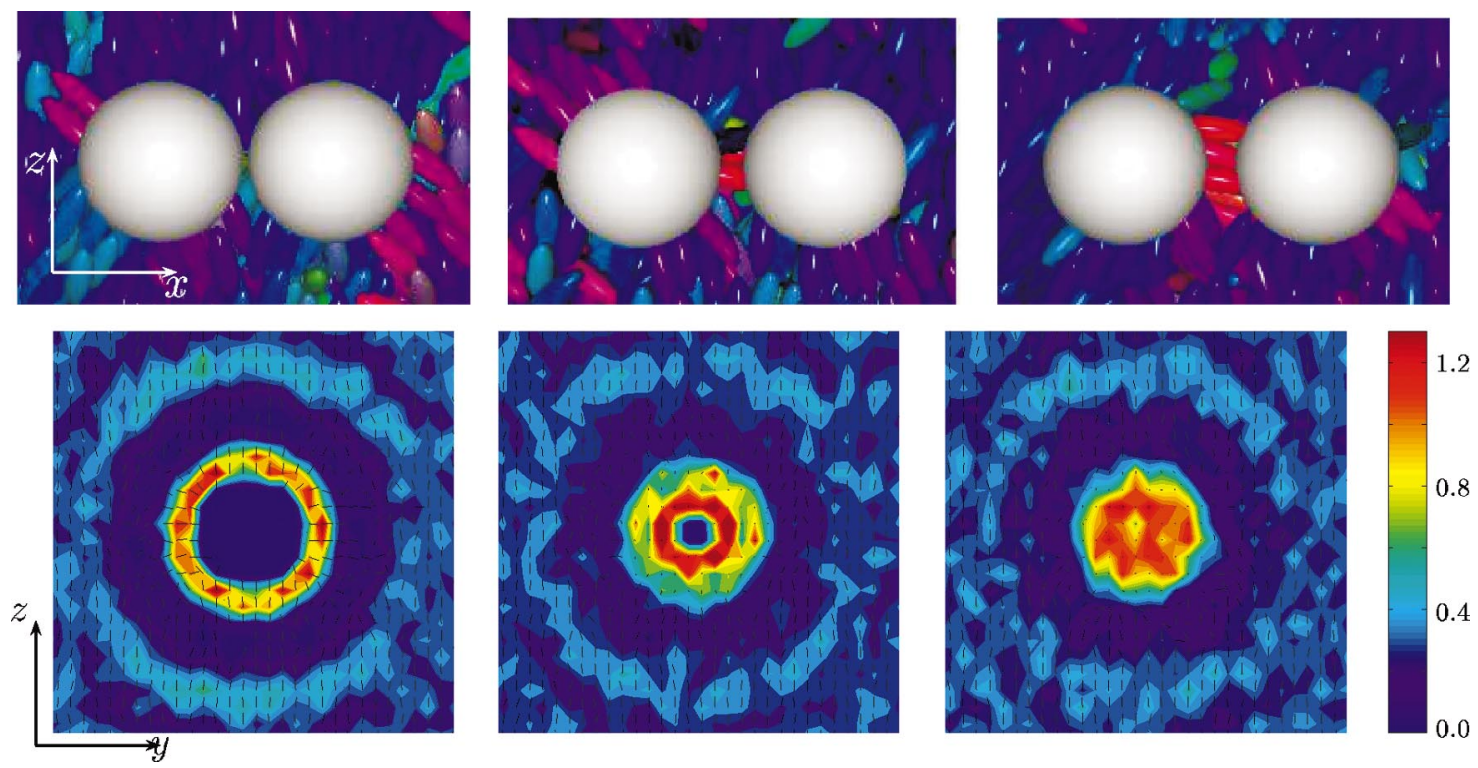

FIG. 5. (Color) Snapshots of the molecules in the vicinity of the spheres separated by $\zeta$ (left to right: $\zeta=0.1,0.78,1 \sigma_{0}$ ) and the corresponding density/director profiles in the midplane perpendicular to the axis connecting the spheres' centers of mass.

character caused by the layered structure of the liquid crystal confined by two homeotropic interfaces. ${ }^{13}$ Since the aligning surfaces of the spheres are finite (having a size comparable to the molecular dimensions), they only induce a small number of (weak) density oscillations. Hence, the amplitude and number of the force and PMF oscillations are smaller compared to the sphere/substrate system.
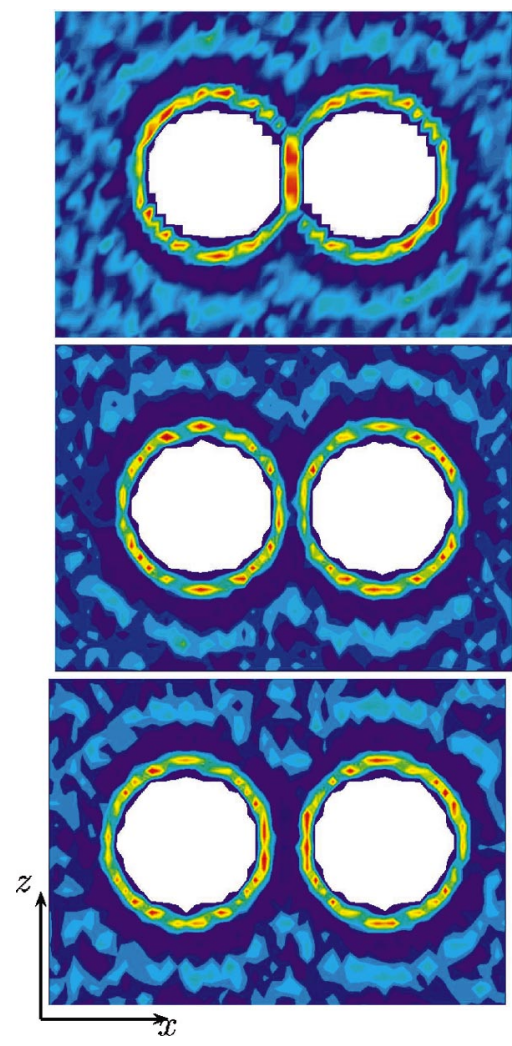

FIG. 6. (Color) Density contour maps at (left to right) the minimum (one layer, $\left.\zeta=1 \sigma_{0}\right)$, maximum $\left(\zeta=2.2 \sigma_{0}\right)$, and second minimum (two layers, $\left.\zeta=3.3 \sigma_{0}\right)$ seen in the PMF in the nematic phase.
In the present case, a maximum of two layers can be supported by spheres of size $R=3 \sigma_{0}$. The layers have a tubular shape bridging the space between the spheres. The long dimension of the tube is in the direction of the intersphere vector, which is also the local director orientation. When the spheres move apart, the outermost molecules that belong to the bridging layers are disengaged from the bridging tube by the influence of vertically oriented molecules in the bulk; thus the horizontally aligned tube shrinks in diameter.

Now we turn to the field-theoretic results: the PMF in the nematic phase obtained by DyFT is displayed in Fig. 4; note that distances are plotted in units of the coherence length $\xi$. Each point was calculated solving the evolution equation for fixed colloid separations. The points were fitted to an empirical function of the form $\sum_{i=0}^{2} a_{i} d^{-(2 i+1)}$, which resembles a multipolar expansion. ${ }^{19}$ As is apparent from the graph, colloids are monotonically attracted down to a separation of $\zeta \approx 0.05 \xi=0.24 \sigma_{0}$, where the fitted PMF curve turns up slightly, indicating repulsion between the colloids.

In comparing the previous result to the PMF from molecular simulations, two differences stand out. First, DyFT curves do not display the undulations seen in the corresponding curves from the Monte Carlo simulations-this is not surprising, since density variations are not accounted for in that theory (we have observed a similar effect in the sphere/ substrate system). Second, the short range repulsion in Fig. 4 is much weaker than the one computed by the simulations. One reason for not being able to discern this very short range repulsion is the resolution of the spatial grid in the DyFT computations that can be attained while using the explicit method to solve the evolution equation in a reasonable amount of computational time. In our previous calculations ${ }^{13}$ of the interactions between two spherical colloids in 2D, we could afford much higher resolution due to the dimensionality of the grid and, indeed, were able to observe a weak 
repulsion between the colloids. Nonetheless, even in these calculations, the value of the PMF at zero separation is still negative unlike in the simulation results. As discussed below in this section, the nature of the topological defect predicted by the latter at very short $\zeta$ differs from the one described by the theory and, therefore, we may not expect the same interaction energies in this range. Returning to the previous work on the sphere/substrate system, it may seem interesting that, in that instance, strong repulsion was predicted by both methods; however, the substrate can be pictured as an infinite sphere and hence, both DyFT and MS would be obtaining similar results for binary interactions where at least one of the objects is large (compared to the molecular size).

Next, we investigate how the interactions between the colloids affect the structure of the topological defects arising in the LC. From previous studies we know that a single sphere in this system would be accompanied by a stable Saturn ring defect. ${ }^{23}$ If the spheres are very far apart, each should be surrounded by an identical Saturn ring of characteristic radius $a$; we have already discussed how this characteristic of isolated rings can be used to establish a correspondence between the length scales of $\sigma_{0}$ and $\xi$. We now pose a question of how do these rings interact at close range.

With molecular simulations, we were able to identify three distinct defect topologies: one, as mentioned above, of two separate rings at long range; second, a three-ring structure at intermediate distances; and, finally, the two Saturn rings melted in the contact area. These structures and the approximate values of $\zeta$ at which the topology changes are indicated in Fig. 3; we designate regions with distinct topology as I (two melted rings), II (three rings), and III (separate Saturn rings). In the rest of this section, we examine each defect structure in detail, relating it to the molecular picture.

For concreteness, we place the spheres' centers along the $x$ axis, symmetrically with respect to the origin. Let the spherical poles facing each other be designated $A$ and $B$ for negative and positive $x$, respectively (refer to Fig. 1). Then the distance between poles $A$ and $B$ is $\zeta$ and the vector joining their centers is $\mathbf{d}$. As can be seen from the mean force or PMF profiles (see Fig. 3), the effective interaction between the colloids sets in at $\zeta \approx 6.5 \sigma_{0}$. Below this value, molecular layers anchored near poles $A$ and $B$ are roughly co-aligned with the spheres' center-to-center direction and start "communicating" with each other through the bulk, orienting the intermediate molecules along the intersphere direction $(x$ axis). Note, however, that the original bulk orientation $\hat{\mathbf{n}}_{\text {bulk }}$ is that of the $z$ axis; therefore, there is a competition between these two preferred orientations.

This competition proceeds in two stages. Initially, the bulk LC confined by $A$ and $B$ melts into a disordered phase: $P_{2}$ decreases from the bulk value of 0.8 to 0.4 by $\zeta$ $=3.6 \sigma_{0}$, while the director changes from $\hat{\mathbf{n}}_{\text {bulk }}$ to a poorly defined vector (isotropic phase). Then, at sufficiently small $\zeta$, the bulk molecules between poles $A$ and $B$ are realigned along $\hat{\mathbf{d}}$ and arranged into LC layers characterized by $P_{2}$ of 0.9. As a result, a new disclination ring in the $y z$ plane is formed. This new defect structure is shown in Fig. 7. On the left, we present a 3D visualization of the surface of $P_{2}$ $=0.26$ computed by DyFT, while on the right we show a
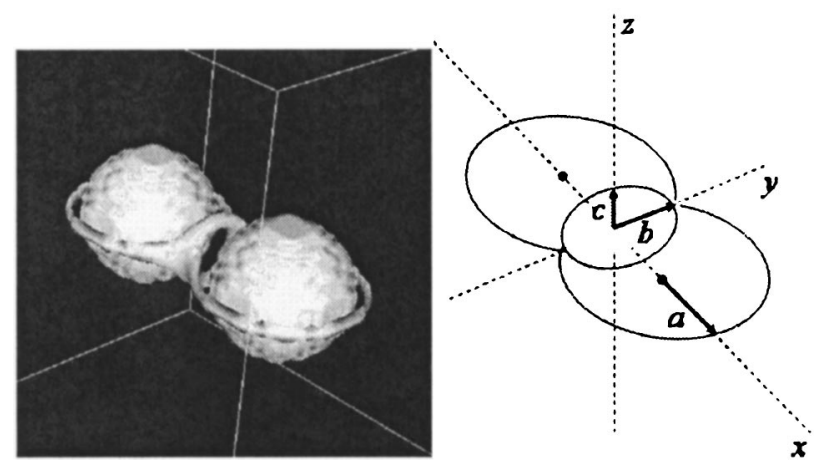

FIG. 7. Contour map of $P_{2}=0.25$ for $\zeta=1 \sigma_{0}$ by DyFT and the corresponding schematic of the defect.

schematic of the defect. The three-ring structure persists throughout region II as indicated on the PMF plot.

When the separation between two spheres becomes smaller than a single-layer thickness, the molecules involved in the layer are gradually displaced out of the tube. Eventually, these molecules reorient radially in directions orthogonal to the intersphere axis, as has been explained above. In this case, the director is discontinuous only in the equatorial plane; the third ring in the $y z$ plane disappears and hence, we observe in effect two Saturn rings fused in one $\infty$-shaped figure.

To compare the theoretical predictions to the molecular picture, we refer to Fig. 8 where profiles of the scalar order parameter along the $x$ axis are plotted for selected values of $\zeta$. (Inside the spheres, the $P_{2}$ profile is arbitrarily shown as zero.) For the sake of comparison, distances are in units of $\sigma_{0}$.

In Fig. 8, the disclinations can be located as the points where $P_{2}(x)$ has a minimum and its derivative is discontinuous. The topmost curve shows $P_{2}$ when the two spheres are sufficiently close for the Saturn rings to interact, and so we

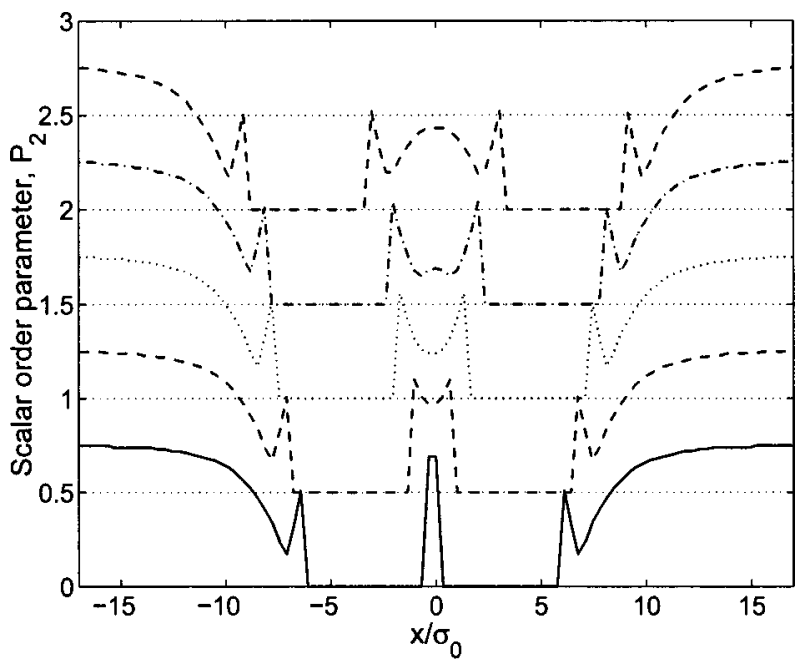

FIG. 8. A series of $P_{2}$ curves along the intersphere axis by DyFT for selected values of $\zeta$ (top to bottom: $\zeta=6.2 \sigma_{0}, 4.2 \sigma_{0}, 3.2 \sigma_{0}, 1.8 \sigma_{0}$, $\left.0.4 \sigma_{0}\right)$; spheres are fixed at $x= \pm \zeta / 2$. Each curve is shifted by 0.5 for clarity. 

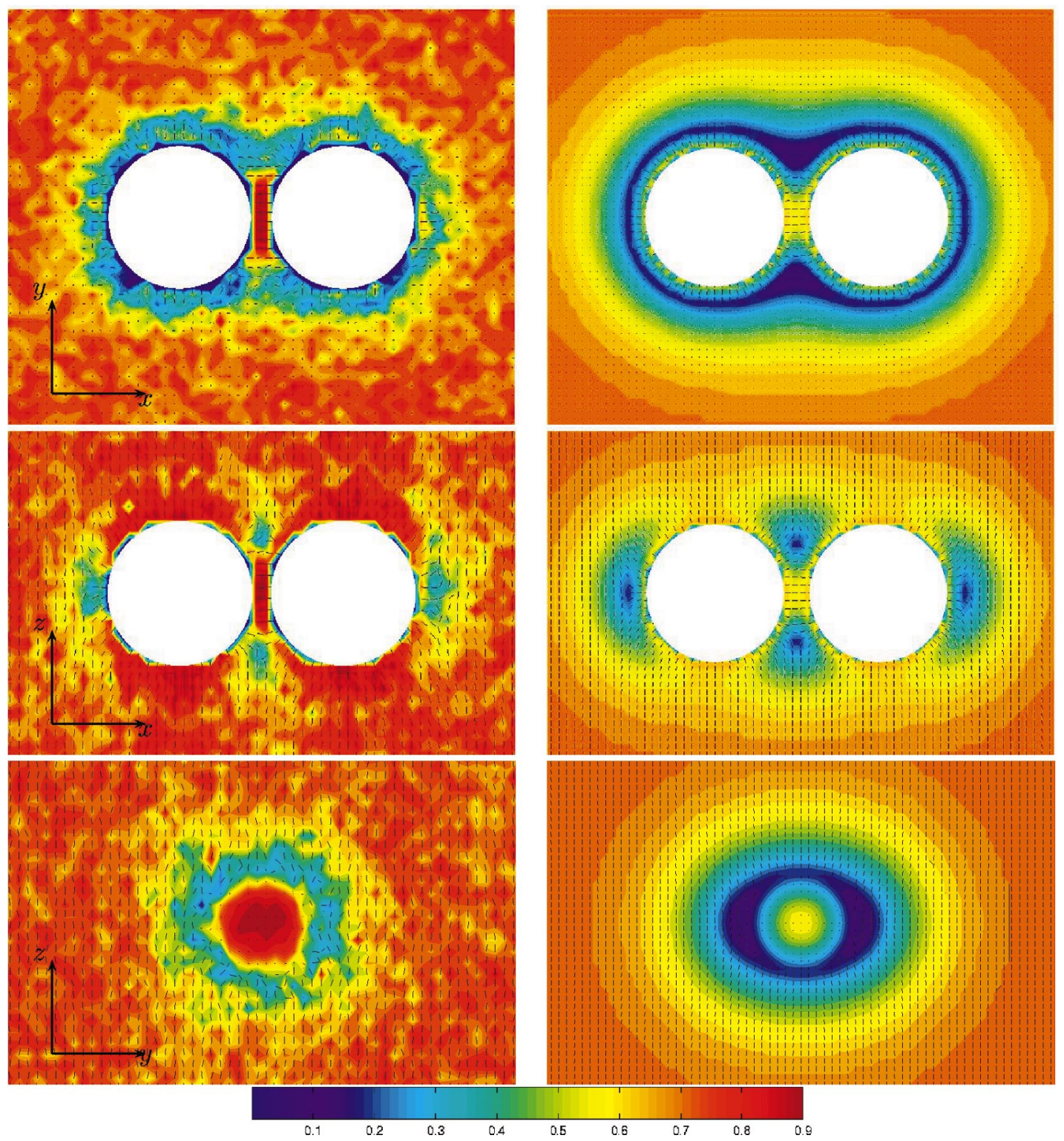

FIG. 9. (Color) $P_{2}$ contour maps with the superimposed director fields at $\zeta=1 \sigma_{0}$ by molecular simulation (left) and DyFT (right) in the $x y, x z$, and $y z$ planes.

observe a region between the inner disclinations where $P_{2}(x)<P_{2}^{\text {bulk }}$. In the absence of interaction, that region would appear as a plateau with $P_{2}(x)=P_{2}^{\text {bulk }}$. Note also that the low value of $P_{2}(x=0)=0.4$ signals the isotropization of the liquid crystal, just as is the case in the molecular simulation. As the spheres continue their approach, the portion of the profiles between each pole and "its" disclination remains unchanged, but the region between the disclinations becomes progressively narrower and disordered. Eventually, at $\zeta$ $=4 \sigma_{0}, P_{2}(0)$ reaches a minimum of 0.2. Simultaneously, the inner disclinations coalesce into a single minimum with a continuous derivative. For still smaller $\zeta$, the value of $P_{2}$ between the poles increases as the spheres get closer, until it reaches values as high as those at the surface near the poles.

We can interpret these $P_{2}(x)$ profiles in the following way: we may consider each sphere surrounded by a region where the local orientation is dictated mainly by the anchoring conditions. When the spheres are apart, there is a gulf where the orientation is dictated by the bulk director. Discli- nations mark places where conflicting regions meet. By bringing the spheres so close that their influence regions overlap, there is no more incompatibility of orientation along the $x$ axis: there will be a tube of director-field lines going from one sphere to the other, and a third disclination ring is formed in the plane orthogonal to the $x$ axis, encircling this tube.

For a direct comparison between simulations and field theory, we plot contour maps of $P_{2}$ with superimposed director fields in three orthogonal planes intersecting at the origin (see Fig. 9). One can see that the results are in excellent agreement overall: both methods predict the same defect topology. The main discrepancy between the two types of maps is that in the MS maps, $P_{2}$ in the molecular bridge is higher than the bulk, whereas in theory, the intersphere value of $P_{2}$ just reaches the bulk value. The latter, however, is a result of the imposed boundary conditions where we set the order parameter at the spheres' surface to equal that of the bulk. 

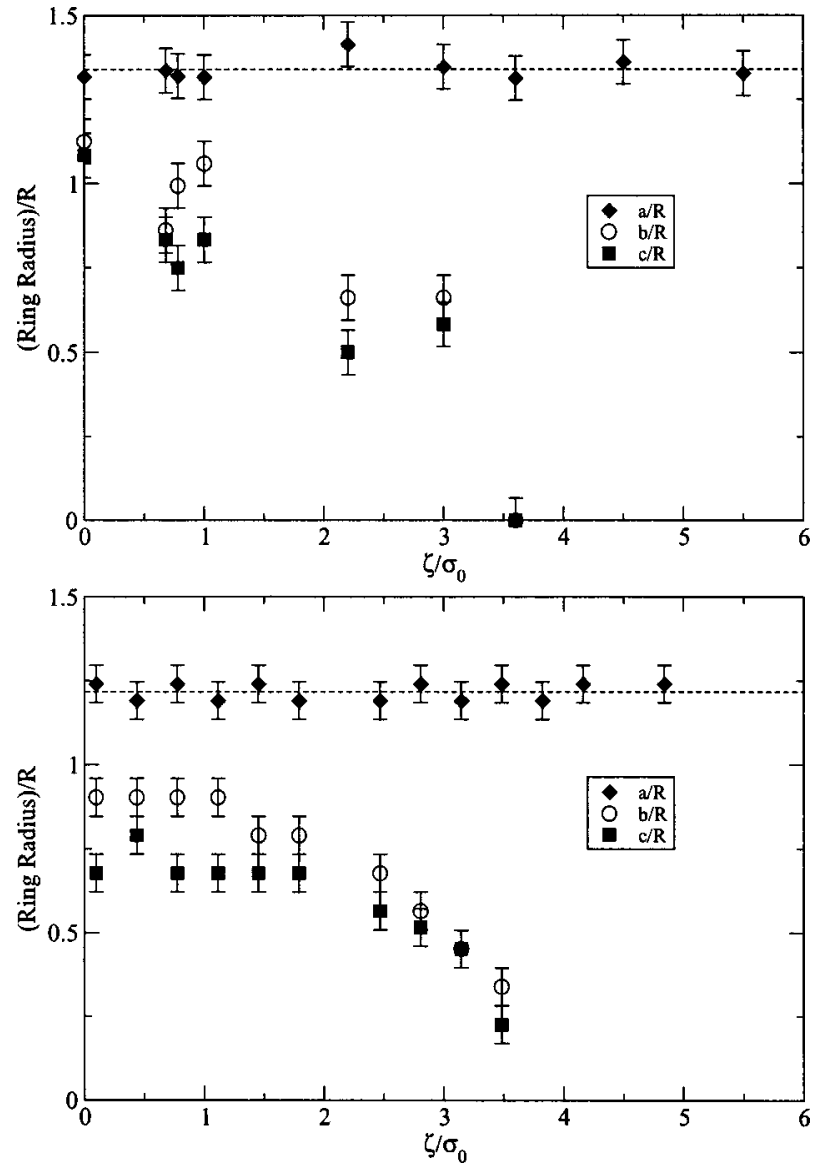

FIG. 10. Dependence of the three principle radii of the three-ring defect on the separation between the spheres by molecular simulation (top) and DyFT (bottom). The lines are a guide to the eye; the error bars reflect the size of the grid.

To further quantify the defect structure, we prepare a plot of the ratios of the three principal radii of the defect to the radius of the sphere, $a / R, b / R$, and $c / R$ (refer to Fig. 7 for definitions) as a function of the spheres' separation; the data are shown in Fig. 10. The ratio $a / R$ describes the radius of the equatorial Saturn rings whether they are fused (short range) or separate (long range). It stays constant at $\sim 1.3$; in fact, this is the only radius that is well defined throughout all the $\zeta$ domain. $b$ and $c$ characterize the size of the third ring in the $y z$ plane; they are defined only in region II for MS, $0.5 \sigma_{0} \leqslant \zeta<3.6 \sigma_{0}$, whereas the third ring is always present in DyFT for $\zeta<3 \sigma_{0}$. The third ring appears elongated along the $y$ axis - this is due to the asymmetry of the system in the $y z$ plane: the bulk director field co-aligned with the $z$ axis reduces the extent of the spheres' aligning field in the $z$ direction. The ring shrinks monotonically as the spheres move away from each other.

\section{B. Colloidal interactions in the isotropic phase}

In this section, we present our results for two spherical colloids dispersed in the isotropic phase obtained by molecular simulations and DyFT. First, we look at the differences of the colloidal interactions between the isotropic $(I)$ and nematic $(N)$ phases; at the same time, we make comparison between the PMF's and defect structures predicted by two methods used in this work; finally, we make a connection between present work and recent experimental and theoretical work on similar systems.

We begin by examining the mean force $F_{\mathrm{sph}}^{I}$ and the PMF in the isotropic phase for $R=3 \sigma_{0}$ by MS shown in Figs. 2 and 3 , respectively. $F_{\text {sph }}^{I}$ has the same shape as the mean force in a nematic $F_{\mathrm{sph}}^{N}$, i.e., the same positions of local maxima and minima; their magnitude, however, is smaller. In particular, $F_{\mathrm{sph}}^{I}$ reaches its maximum at approximately same $\zeta=0.7 \sigma_{0}$ as $F_{\mathrm{sph}}^{N}$, but the maximum value is reduced by a third. This reduction can be attributed to the absence of the bulk director field in the isotropic phase and, hence, no competition between the spheres' aligning field and that of the bulk. Similarly, the attractive force at $\zeta$ $=1.3 \sigma_{0}$ is reduced compared with the $N$ phase. As a consequence, upon integration $F_{\mathrm{sph}}^{I}$ yields a PMF curve that is characterized by a much weaker attractive well than in the $N$ phase $(-2.5 k T$ in the $I$ versus $-15 k T$ in the $N)$; in addition, the minimum in free energy at $\zeta=\sigma_{0}$ is preceded by an energy barrier of $\sim 2.5 \mathrm{kT}$. Therefore, the overall interaction between colloids predicted by the MS is weakly attractive and significantly weaker than that in the $N$ phase.

The oscillatory character of the mean force as well as the PMF is still apparent in the $I$ phase - this is due to the molecules between the spheres packing in a way similar to the $N$ phase. That is, the molecules between poles $A$ and $B$ assemble into layers, thus causing density oscillations; in turn, density oscillations result into an alternating sign of the mean force and in the undulations in the PMF profile. Again, at small separations $\left(\zeta<0.5 \sigma_{0}\right)$ the molecules pack radially with respect to the intersphere axis; at intermediate distances $\left(0.5 \sigma_{0} \leqslant \zeta<3 \sigma_{0}\right)$ a molecular bridge co-aligned with $\mathbf{d}$ is formed; at longer separations each sphere is covered by a homeotropically anchored layer of LC molecules. Snapshots of the system corresponding to the first two regimes are shown in Fig. 5. Since spheres induce layer formation even in the $I$ phase, we may conclude that the chosen interaction potentials (soft repulsive Gay-Berne potential) result in strong homeotropic anchoring. Second, because of the same molecular packing we may expect the same defect structure as seen in the $N$ phase. We must, however, caution against the interpretation of observing a topological defect in the $I$ phase. A topological defect is defined as a region characterized by an abrupt change or discontinuity in the director. In the case of the nematic phase explored in Sec. IV A, the defects are two or three disclination rings surrounded by bulk nematic; in the $I$ phase, the bulklike liquid has an order parameter of zero, and thus, no meaningful preferred orientation. Therefore, the definition of a topological defect does not apply to the structures that we observe in the $I$ phase. Instead, we observe a dumbbell-shaped manifold defined by the order parameter dropping from a nematic nonzero value to an isotropic zero bulk value. A cross section of the dumbbell in the $y z$ plane has an appearance of the third ring seen for the nematic. As an illustration, consider Fig. 11, showing a $P_{2}$ map (with the director field superimposed) for two spheres in the isotropic LC separated by $\zeta=0.78 \sigma_{0}$. The spheres are surrounded by nematic-like coronas due to the presence of well-aligned layers; the nematic tube, aligned 


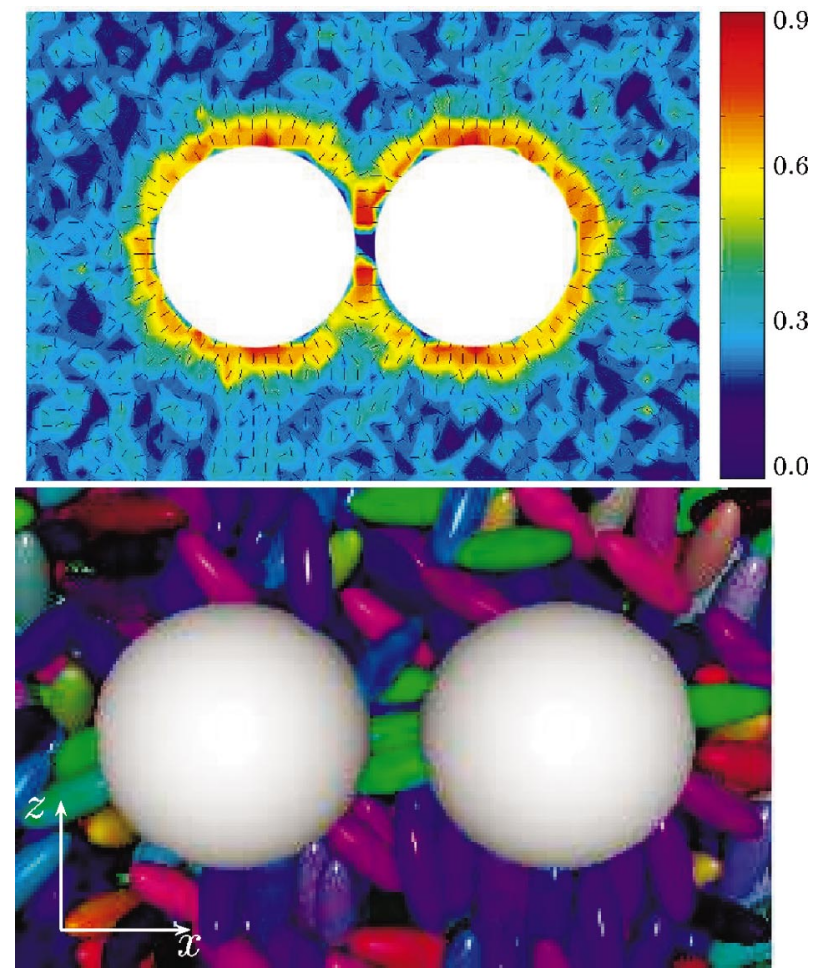

FIG. 11. (Color) Top: Order parameter map with the superimposed director field for two spheres in the isotropic LC at the separation corresponding to the maximum mean force $\left(\zeta=0.78 \sigma_{0}\right)$ in the $x z$ plane. Bottom: Snapshot of the system at the same separation; note that, similar to the nematic phase, the LC layer between the spheres is incomplete.

with the $x$ axis, is already formed and is hollow in the middle. $P_{2}$ is around 0.8 in these areas and the director is well defined; outside of these areas, however, $P_{2}$ drops to a low bulk value of 0.25 and the director appears chaotic and poorly defined. ${ }^{33}$

The interaction between colloids in the $I$ phase computed by $\mathrm{DyFT}^{34}$ is shown in Fig. 4. In this case, we clearly see the overall attraction with the minimum of free energy located at $\zeta=1.5 \xi$; at short range we observe strong repulsion, i.e., the $\mathrm{PMF}$ at contact is positive and has a value of more than twice the attractive well depth. It must be pointed out that the coherence length $\xi$ in the $I$ phase is larger than in the $N$. Therefore, high resolution required by calculations in the $N$ phase is not an issue in this case and we are able to clearly identify repulsion at short range. More importantly, the mapping of the length scales between DyFT and MS no longer applies. Hence, we are not in a position to make a direct comparison between the results from two methods. Instead, we turn to recent work by Galatola and Fournier ${ }^{21}$ who have reported on the interactions between two spherical colloids suspended in the isotropic phase of a nematogenic fluid. Their computations involved analytical solution of a quadratic Landau-de Gennes expansion of the bulk free energy density using spherical harmonics. They were able to map out the phase diagram depending on the size of the colloids and the anchoring strength. According to their predictions, at high anchoring strengths (as is the case presently examined) the spherical colloids will repel each other at short range. We, in fact, observe this type of behavior in the isotropic case. In addition, they identified a typical ring structure arising between two particles at short separations. The size of the ring in the plane perpendicular to the axis connecting the spheres' centers of mass (or midsection of the $P_{2}=0$ manifold) increases linearly with separation-again, we have observed the same effect in the DyFT calculations. The agreement between these two sets of results is not necessarily trivial. As we have seen, in the case of strong homeotropic anchoring, nematic-like regions are created between the particles at close range even in the isotropic phase. Therefore, truncation of the Landau-de Gennes free energy to quadratic terms (i.e., linearization of the evolution equation) may not be a good approximation-we have reported nonlinear field rearrangement in the nematic at similar separations (see Sec. IV A). Expansion to higher powers of $\mathbf{Q}$ in this case does not change the results. This may be due to much lower energies involved in creating the "defect" manifold: one side of the manifold is characterized by the isotropic phase with zero order parameter.

Among the recent experimental studies of colloidal interactions in the isotropic phase of LCs, a particularly relevant example is that by Lin et al. ${ }^{8}$ They performed experiments where optical tweezers were used to control the colloidal spheres in suspensions of rodlike fd bacteriophage virus. The PMFs were measured as a function of rod concentration and the ratio of sphere radius to rod length; the rod concentrations were well below the IN transition. They have observed monotonic attraction of the colloids, which was well described by a depletion potential derived by Yaman and co-workers. ${ }^{35}$ It must be pointed out that the mesogen geometry is dramatically different from that of a Gay-Berne ellipsoid: the fd virus is truly a thin rod with a length-todiameter ratio of $880 \mathrm{~nm} / 6.6 \mathrm{~nm}$. Because of this condition as well as the rods' low concentration, we may not expect similar results. It is, therefore, interesting that a short range repulsion is observed between the colloids at high salt concentration-the latter makes the rods "sticky" at the ends, which results in bridging of the spheres. Bridging may be analogous to a strong anchoring, which, as we have reported, results in strong repulsion for separations smaller than a length of a LC particle. It could be interesting to see if the same experimental methods can be extended to higher rod concentrations or, in other words, the nematic phase.

\section{Size effects on the colloidal interactions in the nematic phase}

In this section, we discuss the effects of the colloid size on the interactions between the spheres in the nematic phase. We also discuss the length and energy scale mapping between the MS and DyFT methods.

As the ExEDOS calculations are computationally intensive, we have relied on our detailed results for spheres of $R=3 \sigma_{0}$ in the $N$ phase to estimate the interactions between the colloids of $R=5 \sigma_{0}$. For that purpose, we ran $20 N V T$ ensemble simulations with the spheres fixed at strategically chosen separations, i.e., corresponding to the extrema in the mean force and PMF profiles for the smaller sphere, to calculate the mean force. We then used piecewise cubic interpolation to obtain a continuous force curve which was sub- 


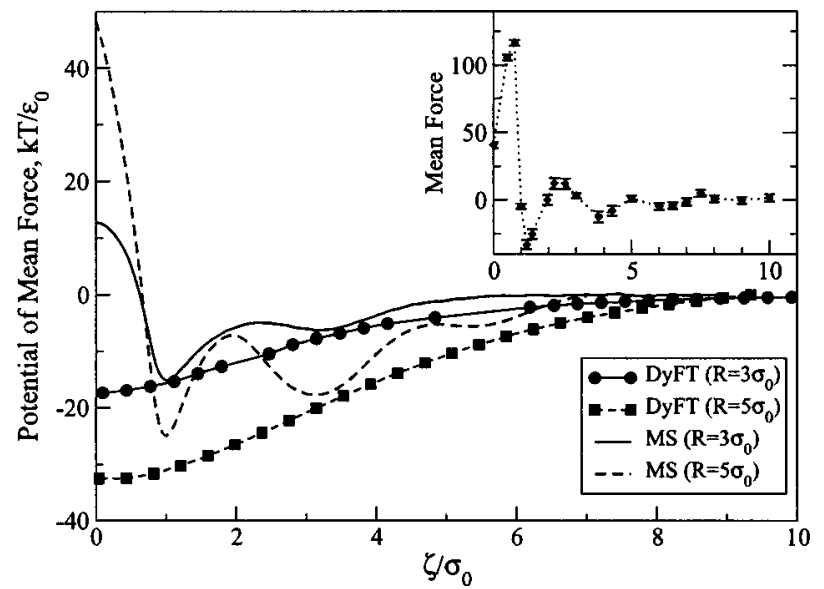

FIG. 12. The PMF for spheres of radius of $3 \sigma_{0}$ and $5 \sigma_{0}$ by DyFT (symbols) and MS (lines). The DyFT free energies are scaled to match the PMFs by MS at $\zeta=1 \sigma_{0}$ (one LC layer formation). The inset: mean force for $R$ $=5 \sigma_{0}$ by MS.

sequently integrated to produce the PMF. The results are plotted in Fig. 12 with corresponding results for smaller spheres for reference. The main effects of the larger size are a much higher repulsive force at short range and the increased amplitude and the number density-driven oscillations in the mean force and the PMF. The increased force and amplitude are to be expected as the size of spherical surfaces surrounding poles $A$ and $B$ is larger and more molecules pack into the layers between them. The strength of the aligning effect of the spheres increases, thus aligning the LC molecules over larger distances and, as a result, forming more LC layers. (Note that, collectively, several spheres induce more layers per sphere than an individual sphere would; for radii of up to $7 \sigma_{0}$, a single layer is anchored by a single sphere.) The overall effects of a larger size are higher repulsion at the distances comparable to a molecular length and greater attractive well depth at $\zeta=\sigma_{0}$. Returning to the analogy with the sphere/substrate system, the effect of stronger attraction with the increasing size for two spheres is the opposite to the "liftoff" of the PMF above the neutral zero value and transition to a purely repulsive interaction.

Using DyFT we ran a series of calculations for spheres ranging from radius of $2.4 \sigma_{0}$ to $17.4 \sigma_{0}$; the resulting PMFs are plotted in Fig. 13. Qualitatively, we observe the same as in the MS size effect: increased well depth and range of the interactions. No significant short range repulsion is observed for all sphere sizes for the same reasons as discussed in Sec. II A. In addition, for the largest size sphere long range repulsion develops for $\zeta>14 \sigma_{0}$. This is consistent with previous studies of an analogous system using the director description. ${ }^{16}$ A common feature of the PMF curves displayed in Fig. 13 is that the slope appears to be nearly constant in the attractive region for a given sphere size; i.e., within certain range, the mean force is independent of separation. At the same time, as shown in the inset, the attractive force in the specified region varies linearly with the spheres' radius.

A similar situation where spherical colloids in an anisotropic solvent were attracted by a constant force was encoun-

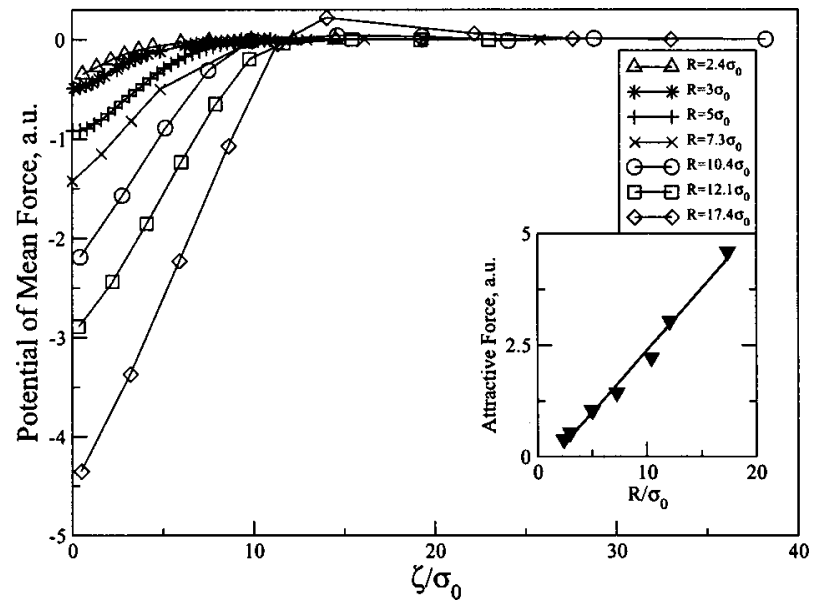

FIG. 13. The PMFs for spheres of radius of $2.4 \sigma_{0}$ to $17.4 \sigma_{0}$ by DyFT. The inset: the attractive force dependence on sphere size.

tered in the experimental setting by Poulin and co-workers. ${ }^{5}$ Two colloids were connected by two birefringent strings in a so-called bubble-gum defect. In this case, the force independent of the separation is a consequence of the free energy being proportional to the length of the disclination lines which, in turn, is set by the colloids' separation. In our calculations, the defect topology is different and more complex. The length of the disclination rings depends on the separation $d$ in a nontrivial way; the same holds for the principal radii of the third ring, $b$ and $c$, as shown in Fig. 10. Therefore, additional contributions to the free energy, other than the energy per unit length of the disclination lines, must be significant in the system considered here. These contributions possibly due to molecular rearrangements would have more weight for small nanoscopic particles attracted along the line perpendicular to the bulk director than for micrometer size colloids interacting along the bulk alignment direction studied by the experiments.

Finally, in order to provide a semiquantitative link between the results of the molecular simulations and field theory we map the energy scales from the two methods. To evaluate the energy scaling coefficient $k^{E}=A / \epsilon_{0}$, we match the PMF for $R=3 \sigma_{0}$ at a separation $\zeta=\sigma_{0}=0.14 \xi$ which corresponds to the global minimum for the MS curve. (At that point, the defect structures from both methods agree with each other.) From this, one obtains the estimate $k^{E}$ $=35$. We then plot the scaled PMF data obtained by the theory for $R=5 \sigma_{0}$ against the corresponding data obtained by simulations in Fig. 12. Taking into account the roughness of the MS estimate of the PMF for $R=5 \sigma_{0}$, the overall agreement is satisfactory.

\section{CONCLUSIONS}

We have investigated the behavior of a system consisting of a confined liquid crystal and two spherical colloids by Monte Carlo simulations and dynamic field theory. We observe a qualitative agreement between the two approaches in the results for the free energy as a function of the spheres' 
separation and the resulting topological defects. We have identified a new three-ring defect structure that arises for this system.

In molecular simulations we see that the layered structure of the LC near surfaces profoundly influences the mean force and the potential of mean force between the spheres at a short range (a few molecular lengths). The PMF minima occur when the spacing between the spherical surfaces is such that an integer number (up to two in the case of the smallest sphere) of LC layers of characteristic thickness can be hosted. Once the spheres cannot support the layers shared by their closest surfaces, the interaction between them ceases and the PMF and mean force curves become featureless. Both methods indicate the presence of short-range repulsion; however, the extent of repulsion predicted by molecular simulations is higher than that computed by the theory. MS results indicate that significant molecular rearrangements at separations comparable to the molecular size alter the defect topology from three rings to two fused Saturn rings. In contrast, the defect topology remains unchanged in the theory. One should not expect agreement in the short-range interactions from the two approaches. In order to observe the layering of the LC molecules, one must incorporate density variations and packing into the dynamic field theory.

In addition, we investigated the effect of the colloids' size on the interactions. We find that the depth of the attraction increases with the size of the spheres. Moreover, the spheres experience a force independent of their separation in the attractive region of the PMF. The magnitude of this attractive force increases linearly with the particle's radius. For the largest spheres considered in this work, a long range repulsion previously reported by the continuum studies precedes the attractive interactions.

Finally, the observation of a new three-ring defect attests to a nontrivial reorganization of an anisotropic medium at the molecular as well as the mesoscopic level. Therefore, for the conditions considered in this work, i.e., nanoscopic particles interacting at distances on the order of a few molecular lengths, a linear superposition of director or tensor order parameter fields produced by each particle is insufficient. Nonlinear terms, such as in the dynamic field theory of $\mathbf{Q}$, must be allowed for. Despite certain limitations in the theoretical results observed at very short range $\left(\zeta<\sigma_{0}\right)$, DyFT provides a powerful tool to study this type of systems, particularly for modeling the mesoscopic behavior of a multiparticle sensor. In general, we find good agreement between DyFT and MS over the large-separation range and also as the size of the colloids increases.

\section{ACKNOWLEDGMENTS}

This work has been supported by the University of Wisconsin Materials Research Science and Engineering Center on Nanostructured Materials and Interfaces (Grant No. NSF-DMR-0079983).

${ }^{1}$ V. K. Gupta, J. J. Skaife, T. B. Dubrovsky, and N. L. Abbott, Science 279, 2077 (1998).

${ }^{2}$ J. A. Van Nelson, S.-R. Kim, and N. L. Abbott, Langmuir 18, 5031 (2002).
${ }^{3}$ P. G. de Gennes and J. Prost, The Physics of Liquid Crystals, 2nd ed. (Clarendon, Oxford, 1993).

${ }^{4}$ Y. Gu and N. L. Abbott, Phys. Rev. Lett. 85, 4719 (2000).

${ }^{5}$ P. Poulin, V. Cabuil, and D. A. Weitz, Phys. Rev. Lett. 79, 4862 (1997).

${ }^{6}$ P. Poulin and D. A. Weitz, Phys. Rev. E 57, 626 (1998).

${ }^{7}$ R. Verma, J. C. Crocker, T. C. Lubensky, and A. G. Yodh, Phys. Rev. Lett. 81, 4004 (1998).

${ }^{8}$ K.-H. Lin, J. C. Crocker, A. C. Zeri, and A. G. Yodh, Phys. Rev. Lett. 87, 088301 (2001).

${ }^{9}$ J. L. Billeter and R. A. Pelcovits, Phys. Rev. E 62, 711 (2000).

${ }^{10}$ D. Andrienko, G. Germano, and M. P. Allen, Phys. Rev. E 63, 041701 (2001).

${ }^{11}$ D. Andrienko, M. P. Allen, G. Skacej, and S. Zumer, Phys. Rev. E 65, 041702 (2002).

${ }^{12}$ E. B. Kim, R. Faller, Q. Yan, N. L. Abbott, and J. J. de Pablo, J. Chem. Phys. 117, 7781 (2002).

${ }^{13}$ S. Grollau, E. B. Kim, O. Guzmán, N. L. Abbott, and J. J. de Pablo, J. Chem. Phys. 119, 2444 (2003).

${ }^{14}$ O. Guzmán, E. B. Kim, S. Grollau, N. L. Abbott, and J. J. de Pablo, Phys. Rev. Lett. 91, 235507 (2003).

${ }^{15}$ E. M. Terentjev, Phys. Rev. E 51, 1330 (1995).

${ }^{16}$ R. W. Ruhwandl and E. M. Terentjev, Phys. Rev. E 55, 2958 (1997).

${ }^{17}$ H. Stark, J. Stelzer, and R. Bernhard, Eur. Phys. J. B 10, 515 (1999).

${ }^{18}$ A. Borstnik, H. Stark, and S. Zumer, Phys. Rev. E 60, 4210 (1999).

${ }^{19}$ B. I. Lev, S. B. Chernyshuk, P. M. Tomchuk, and H. Yokoyama, Phys. Rev. E 65, 021709 (2002).

${ }^{20}$ M. Tasinkevych, N. M. Sylvestre, P. Patricio, and M. M. Telo da Gama, Eur. Phys. J. B 9, 341 (2002).

${ }^{21}$ P. Galatola and J.-B. Fournier, Phys. Rev. Lett. 86, 3915 (2001).

${ }^{22}$ P. Galatola, J.-B. Fournier, and H. Stark, Phys. Rev. E 67, 031404 (2003).

${ }^{23}$ S. Grollau, N. L. Abbott, and J. J. de Pablo, Phys. Rev. E 67, 011702 (2003).

${ }^{24}$ J.-B. Fournier and P. Galatola, Phys. Rev. E 65, 032702 (2002).

${ }^{25}$ A. N. Beris and B. J. Edwards, Thermodynamics of Flowing Systems (Oxford University Press, Oxford, 1994).

${ }^{26}$ F. Calvo, Mol. Phys. 100, 3421 (2002).

${ }^{27}$ L. A. T. Espinoza, K. R. Schumann, Y. Y. Luk, B. A. Israel, and N. L. Abbott, Langmuir 20, 2375 (2004).

${ }^{28}$ N. Schopohl and T. J. Sluckin, Phys. Rev. Lett. 59, 2582 (1987).

${ }^{29}$ Simulations were performed with $A=1, L_{1}=0.55, D^{*}=0.35$. For a nematic potential $U=3$, these values imply $S^{\mathrm{eq}}=0.5, \xi=1.81$, and $\Gamma=0.622$. Given suitable pressure, length, and time scales, these parameters can be mapped to $L_{1}=8.73 \mathrm{pN}$ and $\Gamma=6.22 \mathrm{~Pa}^{-1} \mathrm{~s}^{-1}$. The corresponding Frank elastic constants are then given by $K_{11}=K_{22}=K_{33}=4.37 \mathrm{pN}$. These material parameter values are representative of a $5 \mathrm{CB}$ liquid crystal.

${ }^{30}$ M. Doi and S. F. Edwards, The Theory of Polymer Dynamics (Clarendon, Oxford, 1989).

${ }^{31}$ Simulations were performed with $A=1, L_{1}=0.55, D^{*}=0.35$. For the isotropic and nematic phases we used $U=1.0$ and $U=4.8$, respectively.

${ }^{32}$ E. B. Kim and J. J. de Pablo, Phys. Rev E 69, 061703 (2004).

${ }^{33}$ For the isotropic phase, $P_{2}$ should be zero; however, in a simulation of finite number of particles and over a finite time, this value is virtually unattainable from purely statistical point of view (for a large number of samples $N$ of random orientation, the asymptotic value of the order parameter will be proportional to $N^{-1 / 2}$ ). Therefore, the $P_{2}$ value obtained in a bin on a rectangular grid from only 500 configurations will depend on the bin size, i.e., although the local average $P_{2}$ in a bin far away from the spheres will be representative of any such bin, this value will drop as the bin size increases. For example, if we calculate $P_{2}$ averaged over all configurations and the entire system exclusive of the spheres and the adjacent regions, the resulting value is 0.03 ; for a bin size of $0.4 \times 0.4$ $\times 0.5 \sigma_{0}^{3}$ (the resulting $P_{2}$ map is shown in Fig. 11), the average is 0.25 . Clearly, the latter value is too high; however, our motivation for such fine grid was to reproduce the $P_{2}$ map close to the spheres surface where the ordering changes very quickly with the distance.

${ }^{34}$ To model strong anchoring conditions in the isotropic phase we used the same boundary conditions as in the nematic phase, i.e., a uniaxial order parameter tensor with $U=4.8\left(S^{\mathrm{eq}}=0.8\right)$. In the bulk the nematic potential $U$ was set to 1.0 , which corresponds to the scalar order parameter of zero.

${ }^{35} \mathrm{~K}$. Yaman, C. Jeppensen, and C. M. Marques, Europhys. Lett. 42, 221 (1998). 